\title{
Trans-subxiphoid robotic surgery for anterior mediastinal disease: an initial case series
}

\author{
Osbert Qi Yao Leow ${ }^{1}$, Chuan Cheng ${ }^{2}$, Yin-Kai Chao ${ }^{2}$ \\ ${ }^{1}$ Department of Surgery, Chang Gung Memorial Hospital-Linko, Taoyuan; ${ }^{2}$ Division of Thoracic Surgery, Chang Gung Memorial Hospital-Linko, \\ Chang Gung University, Taoyuan \\ Contributions: (I) Conception and design: OQY Leow, YK Chao; (II) Administrative support: YK Chao; (III) Provision of study materials or patients: \\ YK Chao; (IV) Collection and assembly of data: OQY Leow; (V) Data analysis and interpretation: OQY Leow, YK Chao; (VI) Manuscript writing: \\ All authors; (VII) Final approval of manuscript: All authors. \\ Correspondence to: Yin-Kai Chao, MD. Division of Thoracic Surgery, Chang Gung Memorial Hospital-Linko, 5 Fuxing Street, Taoyuan. \\ Email: chaoyk@cgmh.org.tw.
}

Background: Video-assisted thoracoscopic trans-subxiphoid surgery is an ideal technique for removing anterior mediastinal lesions. The diffusion of this method, however, has been limited by the complexity of surgical maneuvers to be performed in the narrow retrosternal space. Robotic surgery holds promise to overcome the technical limitations of the thoracoscopic trans-subxiphoid approach. Here, we describe a case series of patients who had undergone trans-subxiphoid robotic surgery-with a special focus on short-term outcomes.

Methods: Between January 2018 and January 2019, a total of 20 patients underwent trans-subxiphoid robotic surgery for maximal thymectomy or removal of anterior mediastinal masses. A 3-cm longitudinal incision was performed below the xiphoid process, through which carbon dioxide was insufflated and a camera port was inserted. Subsequently, the lower sections of the mediastinal pleura were detached bilaterally - followed by the creation of two bilateral 1-cm skin incisions on the anterior axillary line in the sixth intercostal space for the insertion of robotic arms. Upon completion of port positioning, the surgical robot was docked.

Results: All robotic surgery procedures were successfully completed. Neither conversion to open surgery nor the creation of additional ports was required. The median operating time and console time were $118 \mathrm{~min}$ [interquartile range (IQR): 84-147 $\mathrm{min}$ ] and $92.5 \mathrm{~min}$ (IQR: 78.5-133.5 min), respectively. Drainage tube positioning was not required in 11 (55\%) patients. There were no operative deaths, and the median length of postoperative hospital stay was 2.5 days (IQR: 2-3 days). One patient had postoperative chylothorax and received conservative treatment.

Conclusions: The results of this case series provide initial support to the clinical feasibility, safety, and short-term positive outcomes of trans-subxiphoid robot-assisted surgery for anterior mediastinal disease.

Keywords: Mediastinal disease; robot-assisted surgery; thymectomy; trans-subxiphoid approach

Submitted Jun 06, 2019. Accepted for publication Jul 10, 2019.

doi: $10.21037 /$ jtd.2019.07.38

View this article at: http://dx.doi.org/10.21037/jtd.2019.07.38

\section{Introduction}

Minimally invasive approaches-accomplished through thoracoscopy or robot-assisted surgery-are gaining momentum for anterior mediastinal operations, including maximal thymectomy in patients with myasthenia gravis (MG) or removal of mediastinal masses (1-3). Compared with traditional trans-sternal resections, minimally invasive approaches cause less tissue trauma and may significantly reduce the risk of surgical complications 
(1-3). Thoracoscopy or robot-assisted anterior mediastinal surgery is generally accomplished through a unilateral approach (4). However, this methodology has inherent limitations, including (I) the difficulty of identifying the contralateral phrenic nerve and (II) the frequent inability to secure an acceptable visual field over the anterior or superior mediastinum.

Recent years have witnessed a growing interest in minimally invasive mediastinal surgery accomplished through a subxiphoid approach-which, differently from the traditional lateral technique-allows obtaining an operative field similar to that achievable via sternotomy (5-7). In addition, soft tissues of the subxiphoid area (differently from the rigid rib structure) allow an easier retrieval of the entire specimen through the surgical incision (8). Unfortunately, trans-subxiphoid thoracoscopic surgery is difficult to implement through conventional non-wristed instruments-with ergonomic discomfort and technical difficulties being major obstacles to the widespread implementation of this technique (5).

Surgical robots equipped with wrist-like joints provide a magnified $(10 \times)$ three-dimensional stereoscopic vision and offer markedly improved ergonomics, ultimately allowing fine dissections even in limited anatomical spaces. We therefore reasoned that robot-assisted surgery may help overcome the technical limitations of the traditional subxiphoid procedure, potentially favoring a more widespread diffusion of this technique. Here, we describe a case series of patients who underwent transsubxiphoid robotic surgery for anterior mediastinal disease. All operations were performed by a single surgeon and the main goal was to investigate the feasibility, safety, and short-term outcomes of this procedure.

\section{Methods}

\section{Study patients}

The study was conducted at the Department of Thoracic Surgery, Chang Gung Memorial Hospital, Linko, Taiwan. We retrospectively reviewed the clinical records of patients who underwent robotic subxiphoid surgery between January 2018 and January 2019. All operations were performed by a single surgeon with expertise in robotic and video-assisted thoracoscopic surgery for removal of anterior mediastinal masses through the lateral approach but without previous experience with the subxiphoid technique.

Patients were evaluated based on chest radiograph, chest computed tomography scan imaging with contrast enhancement and positron emission tomography scan in selected cases. In all patients pre-operative functional assessment consisted of medical history, physical examination, basic blood tests, pulmonary function and cardiologic evaluation with electrocardiogram. Echocardiography and assays of myocardial function were performed in case of a history of heart disease.

Patient inclusion criteria were as follows: (I) diagnosis with of MG or resectable anterior mediastinal tumors and (II) willingness to undergo a partially reimbursed robotassisted operation with a subxiphoid approach. Patients with cardiomegaly, poor cardiac function or severe arrhythmia or presence of advanced disease in need of complex resection and reconstructions were treated with a non-subxiphoid technique.

\section{Pre-docking surgical procedure}

Figure 1 illustrates the key steps of subxiphoid robotic surgery. Upon induction of general anesthesia and intubation with a single-lumen endotracheal tube, we placed the patient in the supine position. The leg end of the operating table was lowered to prevent the contact between the camera head and the patient's body. We performed a $3-\mathrm{cm}$ longitudinal skin incision below the xiphoid process and detached with a finger the adjacent tissues from the back of the sternum in a blinded fashion. A 2- to $3-\mathrm{cm}$ longitudinal incision was subsequently created on the rectus abdominis fascia to allow the insertion of an extra-small wound retractor-with a surgical glove being placed on its outer ring. Upon removal of two glove fingers, two trocars were positioned-with the first being for the thoracoscopic camera and the second for the LigaSure device. After securing the glove fingers to the trocar ends, carbon dioxide was insufflated at an $8-\mathrm{mmHg}$ pressure. The LigaSure device was used to obtain a deeper dissection between the sternum and the anterior mediastinum. The lower sections of the mediastinal pleura were then detached bilaterally. Finally, we performed two bilateral $1-\mathrm{cm}$ skin incisions on the anterior axillary line in the sixth intercostal space, through which two 8-mm trocars for the robotic arms were inserted.

\section{Robotic procedure}

Upon completion of port positioning, a surgical robot (da Vinci Xi model; Intuitive Surgical, Sunnyvale, CA, 


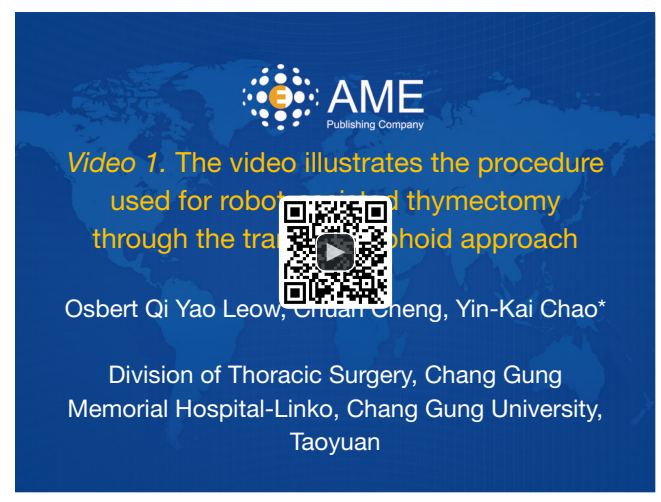

Figure 1 The video illustrates the procedure used for robot-assisted thymectomy through the trans-subxiphoid approach (9). Upon induction of general anesthesia and intubation with a single-lumen endotracheal tube, we placed the patient in the supine position. The leg end of the operating table was lowered to prevent the contact between the camera head and the patient's body. We performed a 3-cm longitudinal skin incision below the xiphoid process and detached with a finger the adjacent tissues from the back of the sternum in a blinded fashion. A 2- to 3-cm longitudinal incision was subsequently created on the rectus abdominis fascia to allow the insertion of an extra-small wound retractor-with a surgical glove (size: $6 \frac{1}{2}$ ) being placed on its outer ring. Upon removal of two glove fingers, two trocars were positioned-with the first being for the thoracoscopic camera and the second for the LigaSure device. After securing the glove fingers to the trocar ends, carbon dioxide was insufflated at an $8-\mathrm{mmHg}$ pressure. The LigaSure device was used to obtain a deeper dissection between the sternum and the anterior mediastinum. The lower sections of the mediastinal pleura were then detached bilaterally (starting from the right mediastinal pleura and subsequently moving to the left mediastinal pleura). Finally, we performed two bilateral 1-cm skin incisions on the anterior axillary line in the sixth intercostal space, through which two 8-mm trocars for the robotic arms were inserted. Upon completion of port positioning, a surgical robot (da Vinci Xi model; Intuitive Surgical, Sunnyvale, CA, USA) was docked from the patient's left side followed by insertion of the robotic camera through the subxiphoid port. The dissecting instruments were introduced and visualized using the robotic camera-with the ProGrasp forceps and the monopolar curved scissor being attached to the left and right arms, respectively. The procedure was started from the right lower pole of the thymus. Excision of the thymus and peri-thymic adipose tissue was accomplished from the pericardiophrenic angle. The insertion of a camera through the midline port facilitates the identification of the thymic upper pole in the cervical region as well as of the bilateral phrenic nerves. Upon its detachment from adjacent tissues, the left lower pole was removed using the same approach (the marks reported here indicate the left phrenic nerve). We subsequently proceeded to the thymic upper pole (with its edge being showed in the video). The right upper thymic pole and the perithymic adipose tissue were resected caudally toward the junction of the innominate vein and the superior vena cava. The left upper pole was cautiously separated from the underlying left innominate vein. An adequate downward traction to the thymus was applied during these procedures. Thymic vessels were sealed with a series of Hemo-lok clips during the detachment of the thymus from adjacent tissues. When all of these procedures were finalized, the extraction of the specimen was accomplished. The subxiphoid approach allowed obtaining a surgical exposure similar to that achievable by sternotomy. The innominate vein and the inferior thyroid vein were both clearly identifiable. We were also able to clearly visualize the aortic arch and its three major branches (i.e., the innominate artery, the left common carotid artery, and the left subclavian artery). Near-infrared fluorescence imaging was applied during surgery. To this aim, a bolus of indocyanine green (ICG) was injected into a peripheral venous catheter. The pericardiophrenic neurovascular bundle was now clearly visible. The specimen (comprising the entire thymus) was finally removed through the subxiphoid port.

Available online: http://www.asvide.com/watch/33090

USA) was docked from the patient's left side-followed by insertion of the robotic camera through the subxiphoid port. The dissecting instruments were introduced and visualized using the robotic camera-with the ProGrasp forceps and the monopolar curved scissor being attached to the left and right arms, respectively.
The procedure was started from the right lower pole of the thymus. Excision of the thymus and peri-thymic adipose tissue was accomplished from the pericardiophrenic angle. We then proceeded to remove the left lower pole using the same methodology. The thymus was thoroughly separated from the underlying left innominate vein, 

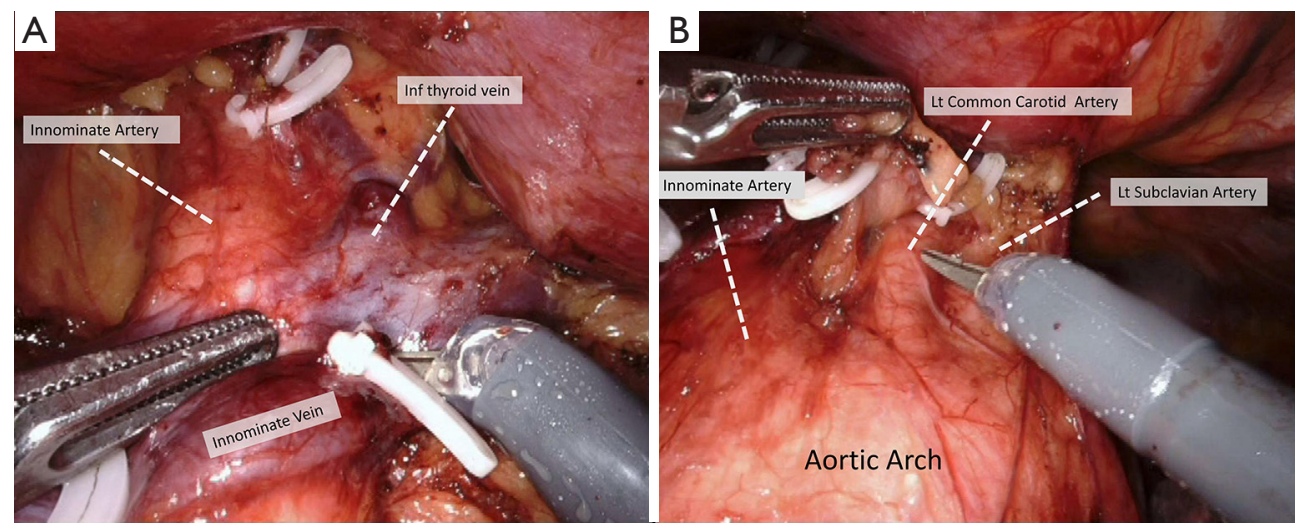

Figure 2 Image obtained after subxiphoid robotic thymectomy. The anatomical landmarks, including the innominate artery/vein (A), the left common carotid, and the subclavian artery (B) were completely skeletonized.

Table 1 Patient and lesion characteristics

\begin{tabular}{|c|c|}
\hline Variable & Value \\
\hline Age, years (median; IQR) & $47.5(39.25-60.25)$ \\
\hline \multicolumn{2}{|l|}{ Sex, n (\%) } \\
\hline Male & $11(55 \%)$ \\
\hline Female & $9(45 \%)$ \\
\hline BMI, kg/m² (median; IQR) & $22.4(20.475-26.45)$ \\
\hline \multicolumn{2}{|l|}{$\mathrm{CCl}, \mathrm{n}(\%)$} \\
\hline 0 & $9(45 \%)$ \\
\hline 1 & $3(15 \%)$ \\
\hline$\geq 2$ & $8(40 \%)$ \\
\hline \multicolumn{2}{|l|}{ Surgical indication, n (\%) } \\
\hline MG without thymoma & $4(20 \%)$ \\
\hline MG with thymoma & $2(10 \%)$ \\
\hline Mediastinal mass without MG & $14(70 \%)$ \\
\hline Tumor size*, cm (median; IQR) & $3.15(1.725-5.75)$ \\
\hline
\end{tabular}

mobilized, and secured with a hemolock clip. After exposure of the superior thymic horns and the inferior portion of the thyroid gland, the upper thymic lobes and the surrounding adipose tissue were dissected in a caudal fashion (Figure 2). Upon completion of the resection, the specimen was removed through the subxiphoid incision. Hemostasis was carefully checked, whereas chest tube drainage was not routinely inserted.

\section{Definition of comorbidities and complications}

The Charlson comorbidity index (CCI) was calculated to assess the severity of comorbidities (10). The total score ranges between 0 and 37, with a score of zero indicating that absence of comorbidities. The Clavien-Dindo (CD) criteria were used to classify the severity of complicationswhich were considered severe in presence of grade IIIa or higher (11).

\section{Statistical methods}

Categorical data are expressed as counts (percentages), whereas continuous variables are given as medians [interquartile ranges (IQR)]. All analyses were conducted with the SPSS statistical software, version 20.0 (IBM, Armonk, NY, USA).

\section{Results}

Between January 2018 and January 2019, 20 consecutive patients (11 men and 9 women; median age: 47.5 years; IQR: 39.25-60.25 years) successfully underwent transsubxiphoid robotic surgery for anterior mediastinal disease. Table 1 shows the general characteristics of the study patients and the excised tumors. CCI scores were as follows: 0 in nine patients (45\%), 1 in three patients (15\%), 2 in five patients (25\%), and 4 in three patients $(15 \%)$. The median body mass index was $22.4 \mathrm{~kg} / \mathrm{m}^{2}$ (IQR: $20.47-26.45 \mathrm{~kg} / \mathrm{m}^{2}$ ). Six patients had a preoperative diagnosis of MG. The 
Table 2 Surgical findings and outcomes

\begin{tabular}{|c|c|}
\hline Variable & Value \\
\hline Total operating time, min [median; IQR] & $118[84-147]$ \\
\hline Robotic console time, min (median; IQR) & $92.5(78.5-133.5)$ \\
\hline $\begin{array}{l}\text { Weight of the surgical specimen, } \\
g \text { [median; IQR] }\end{array}$ & 53 [39-87] \\
\hline \multicolumn{2}{|l|}{ Pathological results, n (\%) } \\
\hline Thymoma & $8(40 \%)$ \\
\hline Thymic cyst & $6(30 \%)$ \\
\hline Thymus hyperplasia & $4(20 \%)$ \\
\hline Teratoma & $2(10 \%)$ \\
\hline \multicolumn{2}{|l|}{ WHO classification of Thymomas, n (\%) } \\
\hline$A B$ & $2(25 \%)$ \\
\hline B1 & $2(25 \%)$ \\
\hline B2 & $2(25 \%)$ \\
\hline B3 & $1(12.5 \%)$ \\
\hline Infarcted & $1(12.5 \%)$ \\
\hline \multicolumn{2}{|l|}{ Masaoka stage of Thymomas, $\mathrm{n}(\%)$} \\
\hline I & $6(75 \%)$ \\
\hline Ila & $2(25 \%)$ \\
\hline Postoperative LOS, days [median; IQR] & $2.5[2-3]$ \\
\hline Chest tube duration, days (median; IQR) & $0(0-1.75)$ \\
\hline \multicolumn{2}{|l|}{ Chest tube drainage $\mathrm{n}(\%)$} \\
\hline No & $11(55 \%)$ \\
\hline Yes & $9(45 \%)$ \\
\hline Conversion to sternotomy, $\mathrm{n}(\%)$ & $0(0 \%)$ \\
\hline \multicolumn{2}{|l|}{ Complications $^{*}, \mathrm{n}(\%)$} \\
\hline No & 19 (95\%) \\
\hline Minor & $1(5 \%)$ \\
\hline Major & $0(0 \%)$ \\
\hline
\end{tabular}

LOS, length of stay; IQR, interquartile range. *, according to the Calvien-Dindo classification.

median tumor size (longest diameter) on CT images was $3.15 \mathrm{~cm}$ (IQR: $1.72-5.75 \mathrm{~cm}$ ).

Intraoperative and postoperative results are summarized in Table 2. The median total operating time and console time were $118 \mathrm{~min}$ (IQR: $84-147 \mathrm{~min}$ ) and $92.5 \mathrm{~min}$ (IQR: 78.5-133.5 min), respectively. All robotic surgery procedures were successfully completed. Neither conversion to open surgery nor the creation of additional ports was required. The median weight of the resected specimens was $53 \mathrm{~g}$ (IQR: $39-87 \mathrm{~g}$ ). The final pathological diagnoses were as follows: thymoma $(n=8 ; 40 \%)$, thymic cyst $(n=6 ; 30 \%)$, thymic hyperplasia $(n=4 ; 20 \%)$, and teratoma $(n=2 ; 10 \%)$. The detailed WHO classification and Masaoka stage of the eight thymoma patients were reported in Table 2. Eleven patients $(55 \%)$ did not require the postoperative placing of chest tube drainage. Two patients with MG underwent postoperative monitoring in an intensive care unit. There were no operative deaths, and the median length of postoperative hospital stay was 2.5 days (IQR: 2-3 days). One patient had postoperative chylothorax (CD grade I) and received conservative treatment.

\section{Discussion}

Despite being considered as an ideal technique for anterior mediastinal lesions, the steep learning curve of videoassisted thoracoscopic subxiphoid surgery has precluded the widespread diffusion of this approach (12). Surgeons who are willing to implement this methodology are not only required to be familiar with thoracoscopic surgery but also need to be skilled in performing the counterintuitive movements typical of this surgery. For example, the dissection of the thymic left lobe requires the grasper forceps to be bent to the right to pull the thymus to the patient's right side-ultimately resulting in a cross-handed movement (6). The dissection of the thymic right lobe is accomplished by bending the grasper forceps to the left with the goal of pulling the thymus to the patient's left side. Under these circumstances, significant interferences between the camera and non-wristed instruments may be a source of major ergonomic discomfort (6).

We believe that the robotic approach offers significant advantages over standard thoracoscopy when the transsubxiphoid technique is performed. First, the robot allows achieving an unparalleled precision when dissections are performed in a limited anatomic space (like the anterior mediastinum). Importantly, tremor-filtration tools and the availability of three-dimensional, magnified operative images markedly reduce the likelihood of accidental injuries to adjacent vital structures. Second, the conventional thoracoscopic subxiphoid approach does not generally allow obtaining a sufficiently large retrosternal working space, especially in patients with large tumor masses and/ or a depressed sternum. In this scenario, the insertion of a sternum lifter (either from the neck or the parasternal 
Table 3 Published studies focusing on robot-assisted thymectomy through the subxiphoid approach

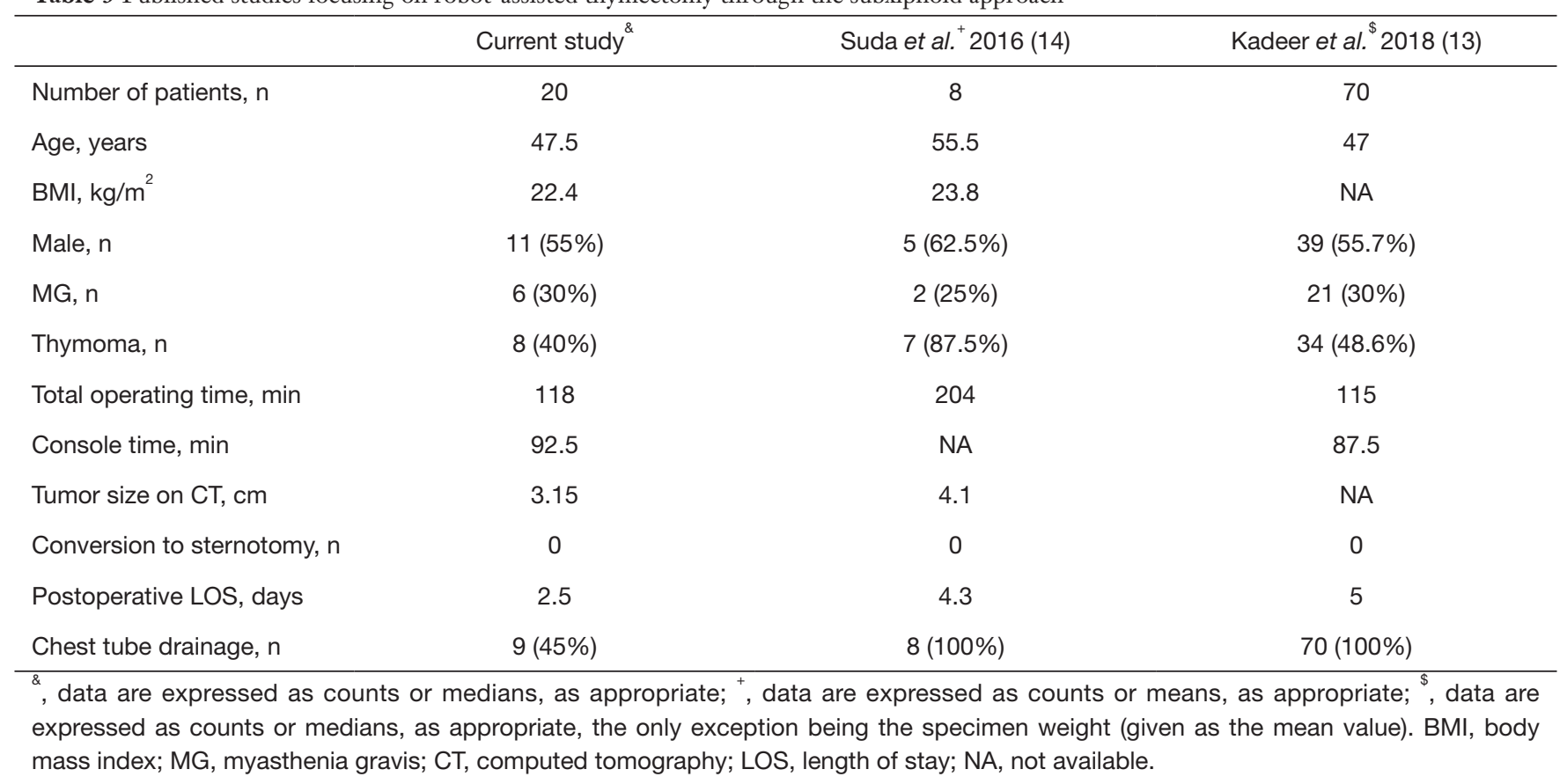

area) may be required to improve surgical exposure. When robotic surgery is used, a moderate lift of the robotic camera arm can produce an effect similar to that achievable with a sternum retractor-without the need of creating an additional wound. Finally, the robotic system allows performing subxiphoid surgery in a more intuitive, ergonomic manner, ultimately abrogating unnatural movements and minimizing the surgeon's fatigue.

Published data on the robot-assisted subxiphoid approach remain limited $(13,14)$. Table 3 summarizes the main results of the two currently available studies. In keeping with previous findings, our case series demonstrates that this robotic procedure is associated with an acceptable procedural time and a negligible conversion rate. Moreover, $55 \%$ of our patients did not require positioning of postoperative chest tube drainage-ultimately shortening the length of hospital stay to less than three days.

Despite the observed favorable outcomes, our findings need to be interpreted in the context of some limitations. Our case series summarizes an initial experience of a single surgeon, and the sample size is limited. Moreover, only patients with non-advanced disease were included in the current report. In addition, our study was not designed to compare the outcomes of the robot-assisted transsubxiphoid approach with the traditional technique. Further studies on a larger number of patients are needed to expand our findings and confirm the feasibility of this technique in patients with mediastinal masses.

\section{Conclusions}

The results of this case series provide initial support to the clinical feasibility, safety, and short-term positive outcomes of trans-subxiphoid robot-assisted surgery for anterior mediastinal disease.

\section{Acknowledgments}

Funding: This study was financially supported by a grant (XPRPG3H0091) from the Chang Gung Memorial Hospital, Taiwan.

\section{Footnote}

Conflicts of Interest: The authors have no conflicts of interest to declare.

Ethical Statement: The authors are accountable for all aspects of the work in ensuring that questions related to the accuracy or integrity of any part of the work are appropriately investigated and resolved. The Institutional Review Board of the Chang Gung Memorial Hospital 
approved the study protocol (CGMH-IRB 201900741B0). The study outcomes will not affect the future patient management. This study is based on data retrieved from a hospital medical record system. All personal data have been protected and secured according to current national and international laws.

\section{References}

1. Roviaro G, Varoli F, Nucca O, et al. Videothoracoscopic approach to primary mediastinal pathology. Chest 2000;117:1179-83.

2. Raza A, Woo E. Video-assisted thoracoscopic surgery versus sternotomy in thymectomy for thymoma and myasthenia gravis. Ann Cardiothorac Surg 2016;5:33-7.

3. Xie A, Tjahjono R, Phan K, et al. Video-assisted thoracoscopic surgery versus open thymectomy for thymoma: a systematic review. Ann Cardiothorac Surg 2015;4:495-508.

4. Marulli G, Rea F, Melfi F, et al. Robot-aided thoracoscopic thymectomy for early-stage thymoma: a multicenter European study. J Thorac Cardiovasc Surg 2012;144:1125-30.

5. Zieliński M, Kużdżał J, Szlubowski A, et al. Transcervicalsubxiphoid-videothoracoscopic "maximal" thymectomyoperative technique and early results. Ann Thorac Surg 2004;78:404-9.

6. Suda T, Sugimura H, Tochii D, et al. Single-port thymectomy through an infrasternal approach. Ann

Cite this article as: Leow OQY, Cheng C, Chao YK. Transsubxiphoid robotic surgery for anterior mediastinal disease: an initial case series. J Thorac Dis 2020;12(2):82-88. doi: 10.21037/ jtd.2019.07.38
Thorac Surg 2012;93:334-6.

7. Lu Q, Zhao J, Wang J, et al. Subxiphoid and subcostal arch "Three ports" thoracoscopic extended thymectomy for myasthenia gravis. J Thorac Dis 2018;10:1711-20.

8. Suda T, Hachimaru A, Tochii D, et al. Video-assisted thoracoscopic thymectomy versus subxiphoid singleport thymectomy: initial results. Eur J Cardiothorac Surg 2016;49:i54-8.

9. Leow OQY, Cheng C, Chao YK. The video illustrates the procedure used for robot-assisted thymectomy through the trans-subxiphoid approach. Asvide 2020;7:050. Available online: http://www.asvide.com/watch/33090

10. Charlson ME, Pompei P, Ales KL, et al. A new method of classifying prognostic comorbidity in longitudinal studies: Development and validation. J Chronic Dis 1987;40:373-83.

11. Dindo D, Demartines N, Clavien P-A. Classification of surgical complications: a new proposal with evaluation in a cohort of 6336 patients and results of a survey. Ann Surg 2004:240:205-13.

12. Yano M, Moriyama S, Haneda H, et al. The subxiphoid approach leads to less invasive thoracoscopic thymectomy than the lateral approach. World J Surg 2017;41:763-70.

13. Kadeer X, Wang L, Zhang L, et al. Modified hook-wire placement technique for localizing multiple pulmonary nodules. J Surg Oncol 2018;118:1188-93.

14. Suda T, Kaneda S, Hachimaru A, et al. Thymectomy via a subxiphoid approach: single-port and robot-assisted. J Thorac Dis 2016;8:S265-71. 